\title{
LOS RECURSOS HÍDRICOS EN EL SISTEMA HIDROGRÁFICO DEL RÍO MIJARES-LA PLANA
}

\author{
J. Quereda Sala, E. Montón Chiva y J. Escrig Barberá \\ Laboratorio de Climatología, Universidad Jaume I
}

\section{RESUMEN}

La Cuenca del Mijares, en el extremo septentrional del Sistema Hidrológico del Júcar, constituye un excepcional ámbito para el estudio de los problemas que plantea la planificación y gestión de los recursos hídricos de la vertiente mediterránea. Al incremento histórico de los regadíos se han sumado el aumento demográfico y el crecimiento industrial, especialmente en la Comarca de La Plana. El presente trabajo desarrolla una metodología para calcular los distintos componentes del balance hídrico y concluir si los recursos satisfacen las necesidades actuales y qué ocurrirá en caso de acontecer un cambio futuro.

Key words: Mijares, recursos hídricos, metodología, balance.

\begin{abstract}
Mijares Basin, at the northern end of Jucar Hydrological System, is an exceptional area to study the problems of planning and management of water resources in the Mediterranean area. The historical increase in irrigation has joined the increasing population and industrial growth, especially in the area of La Plana. This paper develops a methodology for calculating the components of water balance and conclude whether the resources meet current needs and will happen if a future change events.
\end{abstract}

Key words: Mijares, water resources, methodology, balance.

\section{La cuenca del río Mijares: descripción y objetivos del estudio}

Constituye la más septentrional de las tres principales cuencas integradas en el Sistema Hidrográfico del Júcar, siendo la de menor extensión superficial, $4.846 \mathrm{Km}^{2}$, sobre el sistema hidrográfico Mijares-La Plana. El río Mijares, que colecta sus aguas, es el único con caudal permanente, superior a los $10 \mathrm{~m}^{3} / \mathrm{s}$ de módulo, entre las cuencas del Ebro y del Turia. Todo un signo hidráulico de que el río Mijares está alimentado por aguas 
subterráneas. Sin duda alguna la gran excavación llevada a cabo por el río en busca de su nivel de base le ha permitido hacer la captación de los acuíferos subterráneos. El Mijares se erige así en el mayor dren de las aguas que alimentan el sistema hidrogeológico Javalambre-Maestrazgo (Fig. 1).

La red hidrográfica del río Mijares tiene su origen en los derrames mediterráneos del Sistema Ibérico. El amplio anfiteatro de serranías formado por la Sierra de Gúdar y Javalambre sirve simultáneamente de divisoria, a 1.500-2.000 metros, con los ríos AlfambraTuria y con el Guadalope-Ebro. El río nace propiamente sobre los riscos de Gúdar (2.024 m), en términos de Alcalá de la Selva y Cedrillas (Teruel). Las fuentes de cabecera y las precipitaciones de la Sierra de Gúdar, de Javalambre y del Maestrazgo le permiten recoger un caudal apreciable a través de un amplio abanico de afluentes

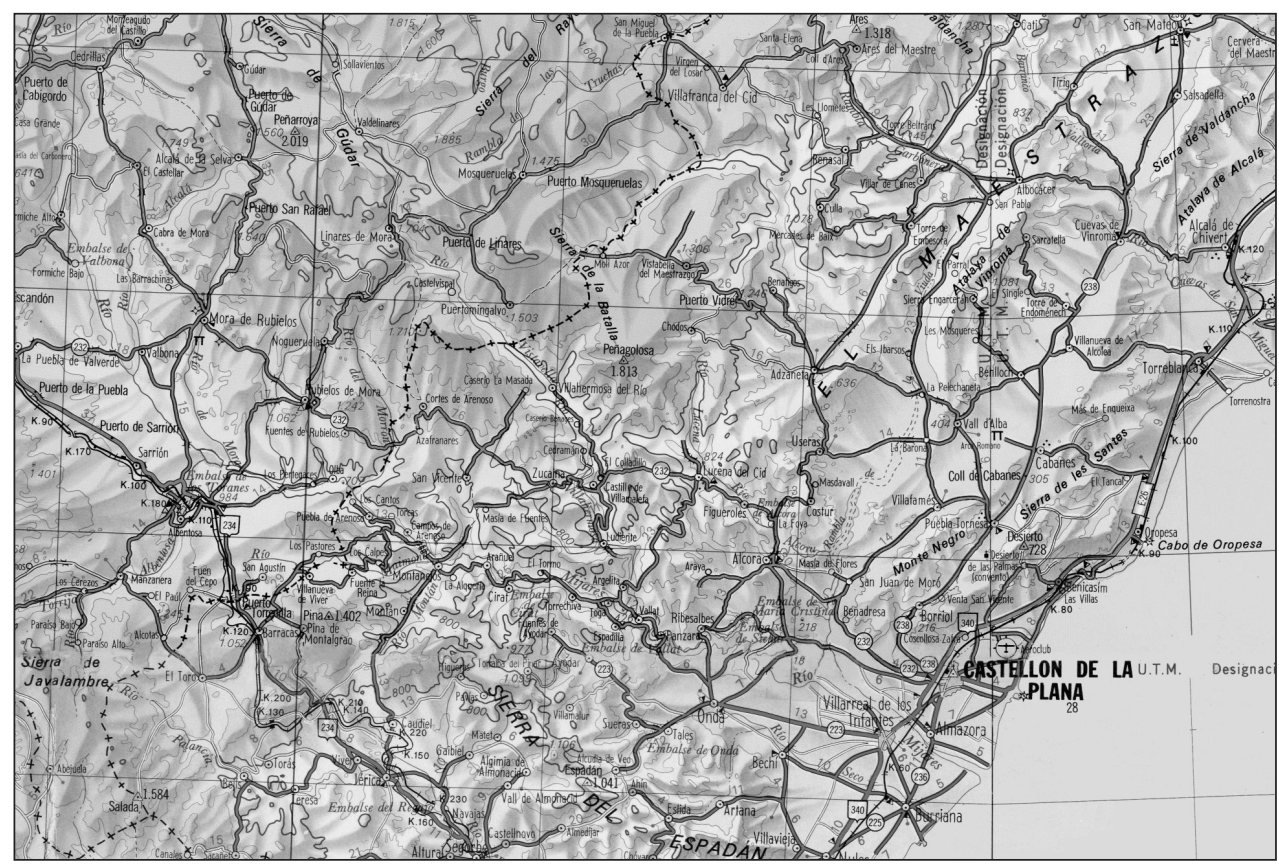

FIgURA 1. El sistema hidrográfico del río Mijares-La Plana, con las cuencas menores de los ríos Belcaire, Seco y Veo, cubre una superficie de $4.846 \mathrm{Km}^{2}$, sobre las provincias de Castellón y Teruel.

El mayor caudal lo aporta el río Albentosa, que desciende desde la Serranía de Javalambre y aporta $3,4 \mathrm{~m}^{3} / \mathrm{s}$ en la fuente de la Escaleruela. Poco antes de esta desembocadura del Albentosa, el río Mijares ha engrosado su caudal con sus dos mayores fuentes, por su izquierda brota la fuente de Babor que aporta $2,7 \mathrm{~m}^{3} / \mathrm{s}$ y enfrentada a ella, en la margen derecha, la fuente de Mas Royo con un aforo idéntico. Puede decirse que estas tres fuentes, con un caudal acumulado próximo a los $9 \mathrm{~m}^{3} / \mathrm{s}$, constituyen el aforo básico del río Mijares (Figs. 2 y 3 ). 


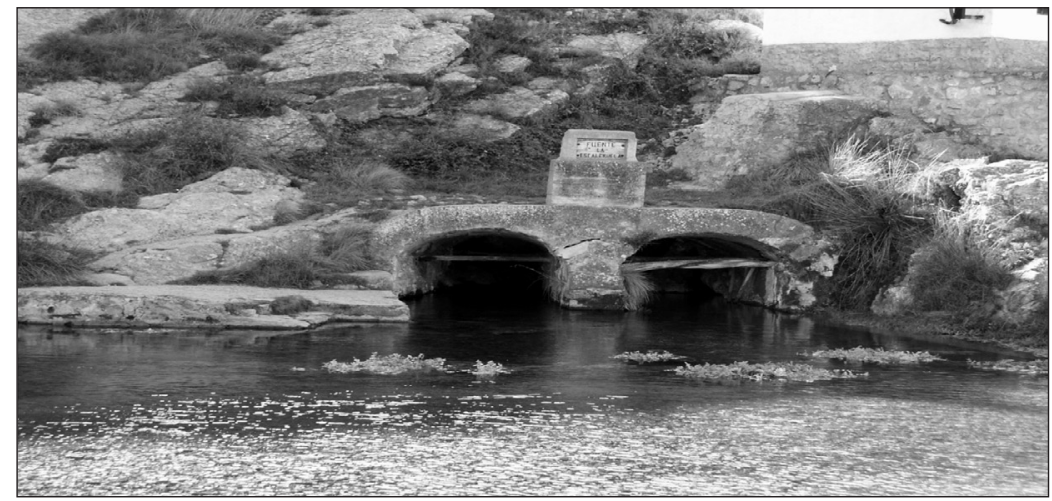

Figura 2. Fuente de La Escaleruela en el río Albentosa, $3,4 \mathrm{~m}^{3} / \mathrm{s}$.

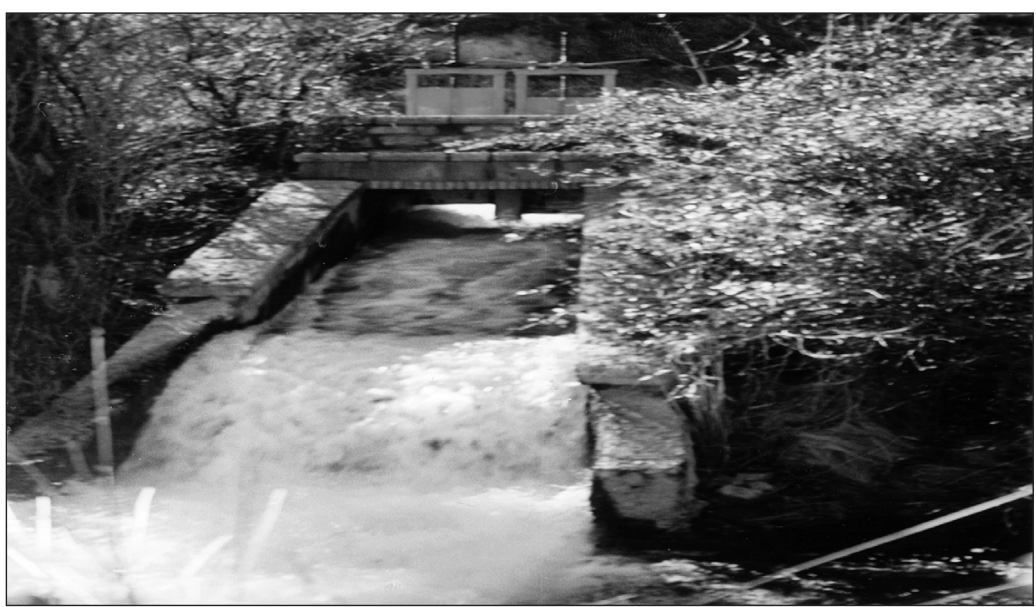

Figura 3. La fuente de Babor en la margen izquierda del río Mijares, unida mediante sifón por debajo del cauce a la fuente de Mas Royo en la margen derecha, en término de Mora de Rubielos, aportan conjuntamente cerca de $6 \mathrm{~m}^{3} / \mathrm{s}$.

Un aforo que, desde mediados del siglo XIV, viene a ser la base hídrica de las regalías otorgadas en los fueros de Jaime I, arbitradas en 1346 por el infante D. Pedro de Aragón, conde de Ribagorza. Esta sentencia constituye la base jurídica del reparto en sesenta filas a las villas de Castellón, Almassora, Vila-Real y Burriana, que fue confirmada por la Ley de Aguas de 1879, artículo 149. Derechos que hoy pertenecen a la Junta de Aguas de La Plana, sociedad que agrupa a los cuatro grandes sindicatos históricos del Mijares, Castellón, Burriana, Vila-Real y Almassora, y que adquirió a fines del XIX los amplios terrenos que rodean las citadas tres grandes fuentes. Unos derechos que han sido perpetuados en las aguas vivas a través del artículo 781 en relación con el 787 del Código Civil (A. GIL OLCINA, 2002). 
La Cuenca del Mijares, situada en esa encrucijada excepcional entre los territorios excedentarios en agua de la Cuenca del Ebro y los ya deficitarios del Júcar, ofrece un ámbito de gran interés para el estudio de los principales problemas que plantea la planificación y gestión de los recursos hídricos en la vertiente mediterránea. Como cualquier otra cuenca hidrográfica, la del río Mijares, con los pequeños ríos Belcaire y Veo, aparece dotada de la independencia hídrica necesaria para poder llevar a cabo el estudio del ciclo hidrológico natural sin aportes exteriores. Un estudio de gran interés en el contexto de la deficitaria situación coyuntural y de la prevista en los actuales modelos de cambio climático, recogida en la reciente planificación hidrológica española.

\section{El déficit hídrico en el sistema hidrográfico Mijares-La Plana y sus causas}

La causa principal de esa rotura de equilibrio entre recursos y extracciones es sin duda el aumento de la superficie regada que se ha venido realizando en toda la cuenca, especialmente intenso en la comarca de La Plana. Una buena expresión de ello es que la superficie regada total se eleva a 43.106 Has en el año 2002 (Censo Agrario), de ellas 41.687, el 97 \%, corresponden a la Provincia de Castellón y 32.439 Has, un $75 \%$ corresponden a la comarca de La Plana (Fig. 4). Una superficie que ha venido aumentando históricamente mediante el aprovechamiento absoluto y celoso de todos los caudales superficiales y subterráneos posibles. Todo ello en un esfuerzo que ya desde sus comienzos tan sólo fue comparable al realizado en Canarias (A. LÓPEZ GÓMEZ, 1957). En los momentos actuales este regadío corresponde en un $41 \%$ al río Mijares y el $59 \%$ al de pozos.

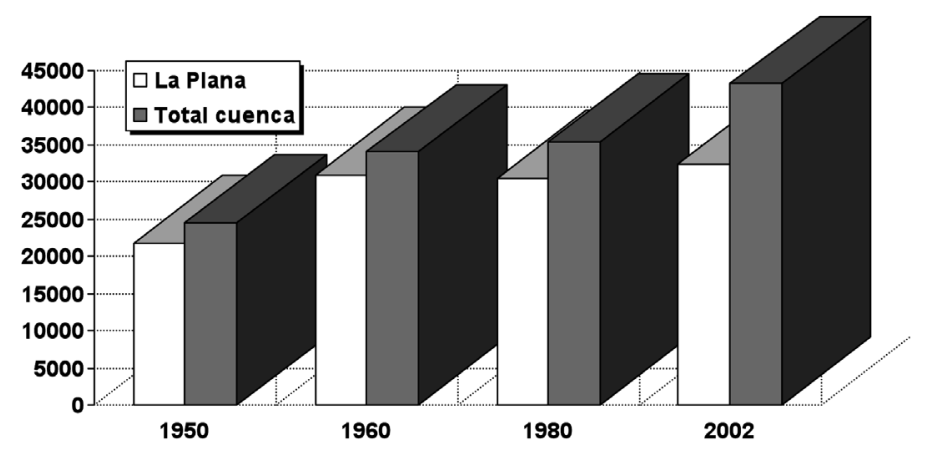

Figura 4. Evolución (Has) de la superficie regada en la Cuenca del Mijares y en la comarca de la Plana durante la segunda mitad del siglo XX.

Este aumento de la superficie regada ha corrido paralelo al aumento demográfico y al crecimiento industrial. La población actual (2010) es de 511.000 habitantes, de los que 502.000 se concentran en la provincia de Castellón, ya que la población turolense del Alto Mijares ha venido descendiendo desde los 40.000 habitantes a comienzos de siglo hasta los 9.000 actuales. Asimismo es un hecho de especial relieve la casi absoluta concentración demográfica que se opera en la comarca de la Plana en la primera década del siglo XXI. En el año 2009, 466.000 habitantes, un $90 \%$ de la población total de la cuenca, se localiza, con características urbanas, sobre el solar comarcal de La Plana (Fig. 5). Este crecimiento demográfico ha sido espectacular, superior al $27 \%$, en la última década (2001-2010). 


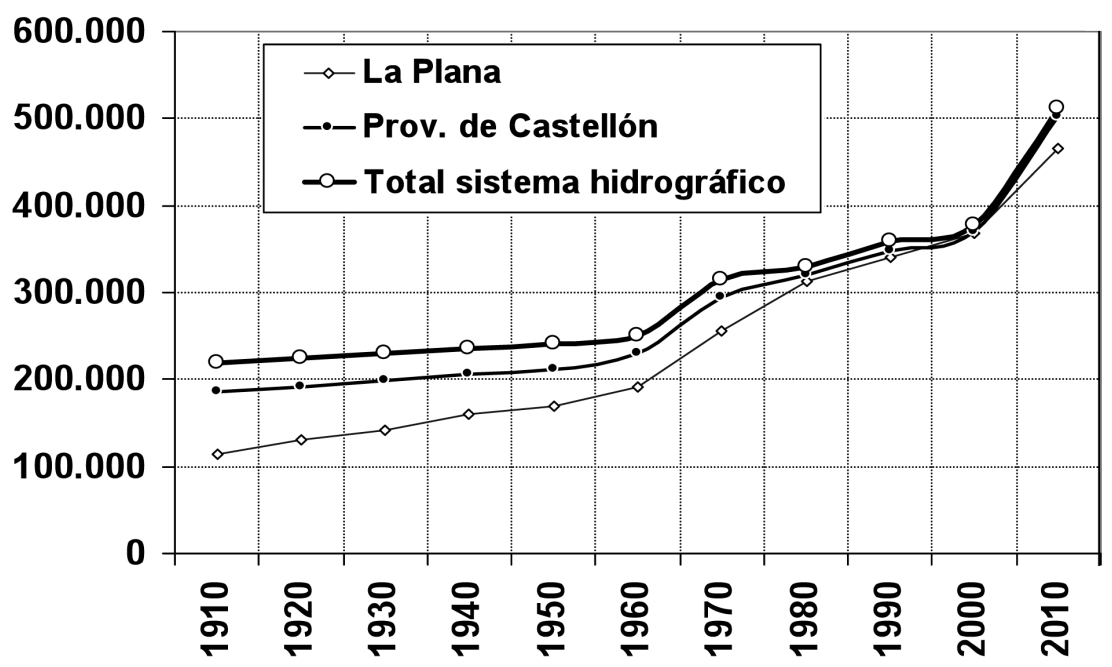

Figura 5. Evolución del número de habitantes en el Sistema Hidrográfico del río MijaresLa Plana.

Este notabilísimo aumento de las demandas ha venido determinando una intensa extracción de recursos hídricos subterráneos traducida, a su vez, tanto en la progresiva reducción de los caudales aforados como en una sobreexplotación y salinización de los acuíferos. Una situación que parece anunciar prematuramente la prevista en la reciente planificación hidrológica. En efecto, el Libro Blanco del Agua en la Comunidad Valenciana (1985), el Proyecto de Directrices (1992), el Plan de Cuenca del Júcar (1999), el Libro Blanco del Agua en España (2000), y el Plan Hidrológico Nacional (2001), sin menoscabo de sus diferencias de estimación, han venido coincidiendo en definir a la Cuenca del Júcar como territorio en situación global de escasez coyuntural. Asimismo se considera que la incorporación del continuado incremento de los consumos y de la recuperación de los acuíferos no solo intensificará el desequilibrio espacial sino, lo que es más preocupante, tornará en claramente deficitario el difícil equilibrio global de recursos-demanda en los próximos años. Un desequilibrio que podría extremarse ante la reducción de recursos hídricos prevista en las bases científicas de los dos últimos informes Third and Fourth Assessment del IPCC (Panel Intergubernamental para el Cambio Climático), Climate Change, 2002 y 2007 de las Naciones Unidas, así como en la reciente Convención del IPCC Valencia (2007), basadas en los modelos del Hadley Centre. Previsiones que marcan los objetivos del presente estudio: la evaluación de los recursos hídricos disponibles y la de su afección por un posible cambio climático.

La evaluación de los recursos hídricos sobre el Sistema Hidrográfico del Mijares-La Plana: metodología de cálculo

La evaluación de los recursos hídricos totales, superficiales y subterráneos, ha sido efectuada mediante la concepción del ciclo hidrológico. En este concepto, los recursos naturales propios son los que se generan a partir de la precipitación y que, subsistentes a la evapotranspiración, nutren y son aportados a través de las escorrentías superficia- 
les directas y la recarga a los acuíferos. Lamentablemente el cálculo de este balance hidrológico, en su régimen natural, es una tarea que ofrece grandes dificultades y que técnicamente todavía no está bien resuelta. En efecto, los datos básicos para este conocimiento deberían estar basados en las medidas de las estaciones de aforos. Unas medidas que contabilizan simultáneamente tanto las aportaciones superficiales como también las subterráneas procedentes de infiltraciones aguas arriba y que en cada punto de la cuenca se incorporan al cauce.

Desgraciadamente los datos de las estaciones de aforos, ademas de escasos, suelen medir regímenes afectados por la actividad humana y no es fácil obtener una información suficiente sobre la evolución de los caudales detraídos o retornados de los ríos en sus diversos usos o afecciones. Todo ello determina que la restitución de los caudales en régimen natural sea una tarea de enormes dificultades (Fig. 6). Ello ha determinado la adopción de una metodología basada en los datos climáticos.

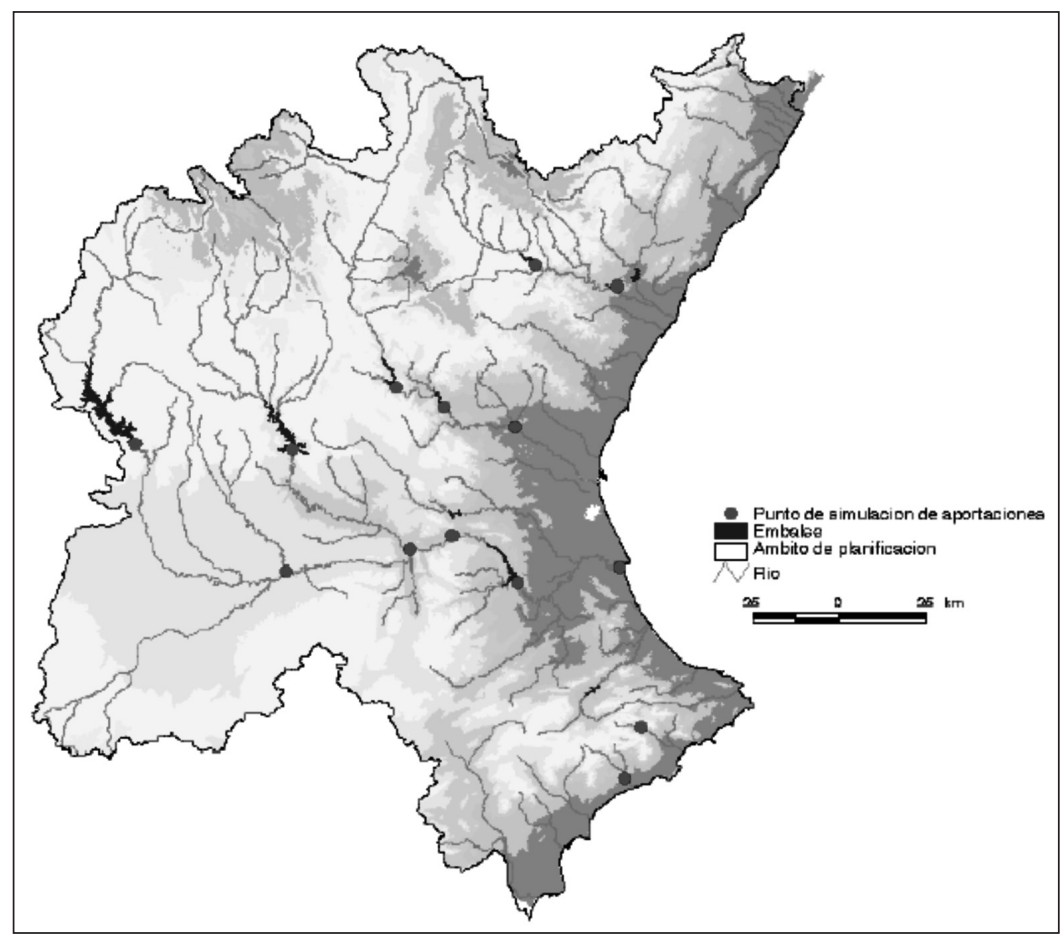

Figura 6. Puntos básicos de evaluación de recursos hídricos en la Cuenca del Júcar (PHN).

En orden de conocimientos y frente a la serie de dificultades que plantean las insuficientes redes de medida superficiales y subterráneas, la red meteorológica puede permitir mejores resultados. En efecto, ante la estrecha dependencia que el ciclo hidrológico mantiene con los datos climáticos, el conocimiento de los principales valores hidrológicos del clima sobre el territorio, nos permite proceder a la estimación del ciclo natural. Esta estimación viene fundamentada en la dependencia que el valor de la escorrentía (E), o aportaciones 
totales anuales (A), muestra con los valores de la precipitación (P) y con los de la evapotranspiración potencial o real (ETP-ETR), procesos estos últimos muy vinculados a la temperatura $(\mathrm{T})$. Este método permite alcanzar buenos resultados. Incluso por definición estos resultados pueden ser óptimos sobre la base de reunir dos condiciones. La primera es que la red meteorológica disponible, con series de suficiente longitud, no presente sesgos de localización que falseen la modelización cartográfica posterior (Fig. 7). La segunda es la medida rigurosa de la evapotranspiración. Condiciones que, debidamente consideradas, pueden haber introducido algún error en nuestro análisis. La malla espacial sobre el sistema hidrográfico del río Mijares, con un observatorio cada $300 \mathrm{Km}^{2}$, no es excesivamente amplia, salvo en algunos territorios como el Bajo Maestrazgo o Penyagolosa.

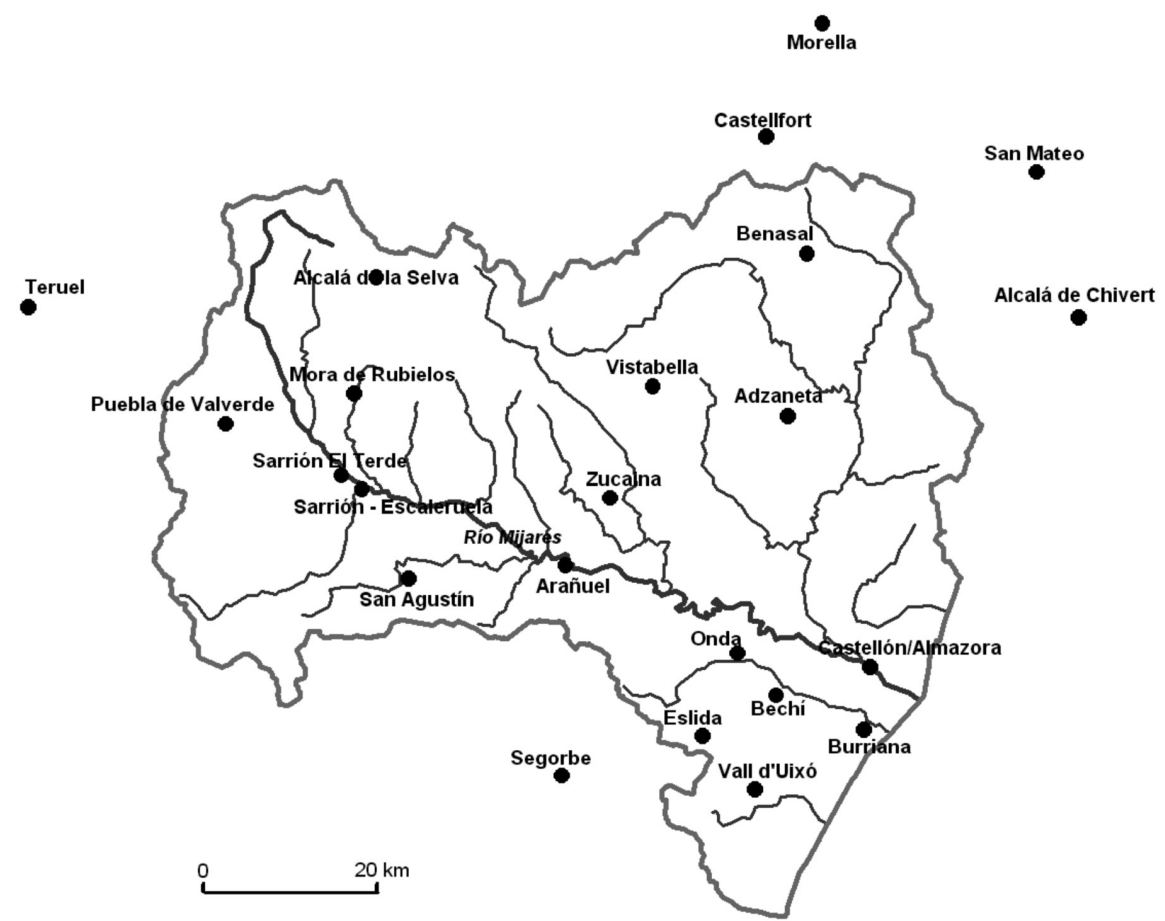

Figura 7. Puntos de observación termopluviométrica utilizados en el análisis. El trazado cartográfico se ha realizado con algunos puntos exteriores a la cuenca para mejor apoyo de las isolíneas.

Esta red meteorológica ha permitido calcular el valor de los recursos hídricos existentes sobre el Sistema Hidrográfico del Mijares mediante la modelización de los elementos climáticos básicos que intervienen en el ciclo del agua. Estos elementos han sido aquellos de cuyas interacciones surge el concepto básico de la escorrentía total o recursos hídricos disponibles (E) o aportaciones en régimen anual (A):

$$
\mathrm{E}(\mathrm{A})=\mathrm{P}-\mathrm{ETR}
$$


La aportación pluviométrica $(\mathbf{P})$. Constituye la fuente por excelencia de las provisiones acuíferas tanto superficiales como subterráneas. De ahí que el análisis pluviométrico haya constituido una preocupación esencial de este estudio. A tal efecto, el fundamento del mismo ha consistido en el establecimiento riguroso de la base de datos de todas las estaciones pluviométricas disponibles (CHJ y SIGA). Los valores registrados en los 66 observatorios pluviométricos han servido para elaborar el mapa territorial de isoyetas medias anuales mediante interpolación de Kriging. Este mapa, mediante gradación cromática y lineal, permite observar que la mayor parte de la cuenca se mantiene por debajo de los $600 \mathrm{~mm}$ anuales (Fig. 8). Las isoyetas anuales medias se hallan entre los $450 \mathrm{~mm}$ de la zona costera y los $800 \mathrm{~mm}$ del macizo de Penyagolosa, Vistabella, sobre la isohipsa de 1.250 metros. Aunque con toda probabilidad y sobre las cimas de Gúdar puedan registrarse valores semejantes a los de Vistabella, las precipitaciones registradas en Alcalá de la Selva, también a 1.250 metros de altitud, son tan sólo de $554 \mathrm{~mm}$. Unas precipitaciones que igualmente se mantienen entre los 500 y los $550 \mathrm{~mm}$ en los observatorios de Puebla de Valverde y Mora de Rubielos situados sobre la isohipsa de 1.000 metros de altitud. Sin duda que ello viene a reflejar el efecto de sombra pluviométrica proyectado localmente por los macizos de Gúdar y Penyagolosa. Globalmente las precipitaciones medias de 556 mm aportan anualmente $2.694 \mathrm{Hm}^{3}$ sobre $\operatorname{los} 4.846 \mathrm{Km}^{2}$ de la superficie total del sistema hidrográfico Mijares-La Plana.

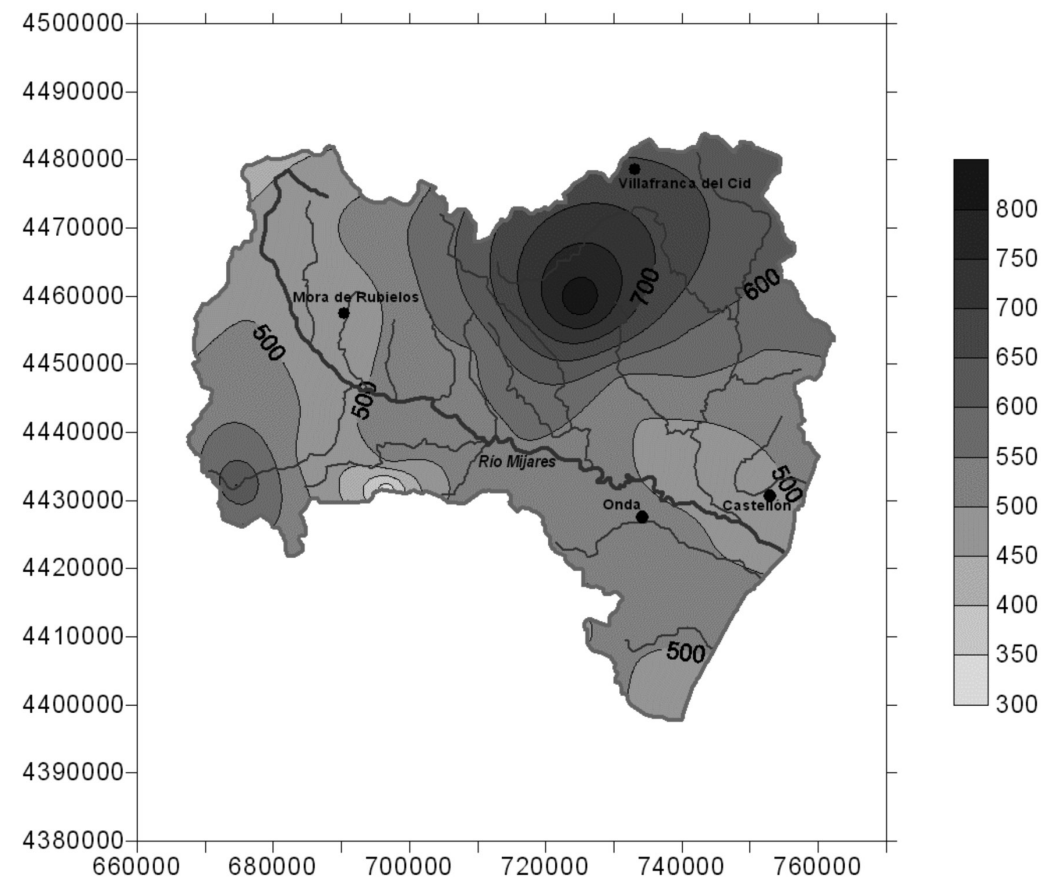

FIgURA 8. Valores de la precipitación media anual sobre el Sistema Hidrográfico del río Mijares-La Plana (1950-2000). 
La evapotranspiración (ETP-ETR). La aportación pluviométrica constituye la fuente por excelencia de las provisiones acuíferas. Sin embargo, los recursos hídricos disponibles no son todos esos volúmenes de agua precipitados, ya que una gran parte de los mismos es consumida en los procesos de evaporación física y biológica. Ello hasta tal punto que las regiones definidas como áridas deben esta característica más a su elevada evapotranspiración que a la escasez de lluvias. De ahí la gran importancia que tiene conocer con la mayor precisión posible la magnitud de la evaporación, el factor clave del ciclo del agua y charnela de todo el proceso.

Esta evaluación de la ETP ha sido llevada a cabo mediante dos métodos: el de Thornthwaite y el de Turc. El primero por su mejor adaptación a los datos meteorológicos existentes y a la planificación hidrológica (PHN), y el de Turc por su mejor concepción para proyecciones de cambio climático.

La fórmula propuesta por Thornthwaite (1948 y 1955) para evaluar la evapotranspiración es enteramente dependiente de la temperatura:

$$
\mathrm{E}=1 \text { ' } 6\left(10 \frac{t}{I}\right)^{a} * f
$$

donde E, es la evapotranspiración potencial mensual en $\mathrm{cm}$; $\mathrm{t}$, la temperatura media mensual en ${ }^{\circ} \mathrm{C} ; f$, el coeficiente para corregir la duración del día, y los coeficientes $I$ y $a$ son constantes de cada estación basadas igualmente en su temperatura. Así el valor I, o coeficiente de calor anual, del que deriva el exponente $a$, es la suma de los doce coeficientes de calor mensuales $i$,

$$
\sum_{i=1}^{12}
$$

calculados a partir de la temperatura media de cada mes $(t)$, mediante la ecuación:

$$
i=\left(\frac{t}{5}\right)^{1 / 514}
$$

La misma vinculación con la temperatura muestra la formulación de Turc para la ETP en mm (1954 y 1961):

$$
\mathrm{ETP}=0^{\prime} 4 \frac{t}{t+15}\left(R s+50\left(0^{\prime} 37\right)\right)
$$

donde $t$ es la temperatura media del mes y Rs es la radiación solar global día en cal. $\mathrm{cm}^{-2}$ (J. QUEREDA, 1982). Este factor se puede calcular mediante

$$
\text { Rs }=\text { RA (0’29 } \cos \phi+0 ’ 54 \mathrm{I})
$$

donde RA es la radiación solar media recibida en el límite de la atmósfera en cal. $\mathrm{cm}^{-2}$ día $^{-1} ; \phi$, la latitud e I, el cociente de insolación.

Los valores mensuales de evapotranspiración potencial obtenidos son más elevados con el método de Turc (1961) que con el método de Thornthwaite. Esta sobreelevación se pone de manifiesto en el hecho de que la medida de Turc es equivalente a la de las experimentales del Piché y de tanque sin corregir (*0’8) (J. QUEREDA y A. CALVO, 1981). 
En consecuencia hemos utilizado los valores de Thornthwaite para el trazado cartográfico de la ETP (Fig. 9). En todo caso es evidente que estas funciones permiten establecer una elevada correlación entre precipitaciones, evapotranspiraciones y escorrentías. No obstante, si bien esta correlación no es discutible, no sucede lo mismo en cuanto a los valores obtenidos, muy dependientes de la formulación empleada (J. QUEREDA, 1976, 1990, 2005, y C. ALMARZA, 1984).

La evapotranspiración potencial (ETP) correspondiente a la cuenca del río Mijares es la mostrada en la figura 9. Altamente tributaria de la temperatura, la ETP alcanza sus mayores valores en las zonas bajas y va reduciéndose a medida que el territorio se eleva. De este modo, la evapotranspiración potencial se mantiene entre valores anuales superiores a $850 \mathrm{~mm}$ sobre la zona litoral y valores algo inferiores a $600 \mathrm{~mm}$ en las tierras altas del Maestrazgo-Gúdar. Globalmente sobre la superficie total de la cuenca, la evapotranspiración potencial demandada por la atmósfera se eleva a $712 \mathrm{~mm}$.

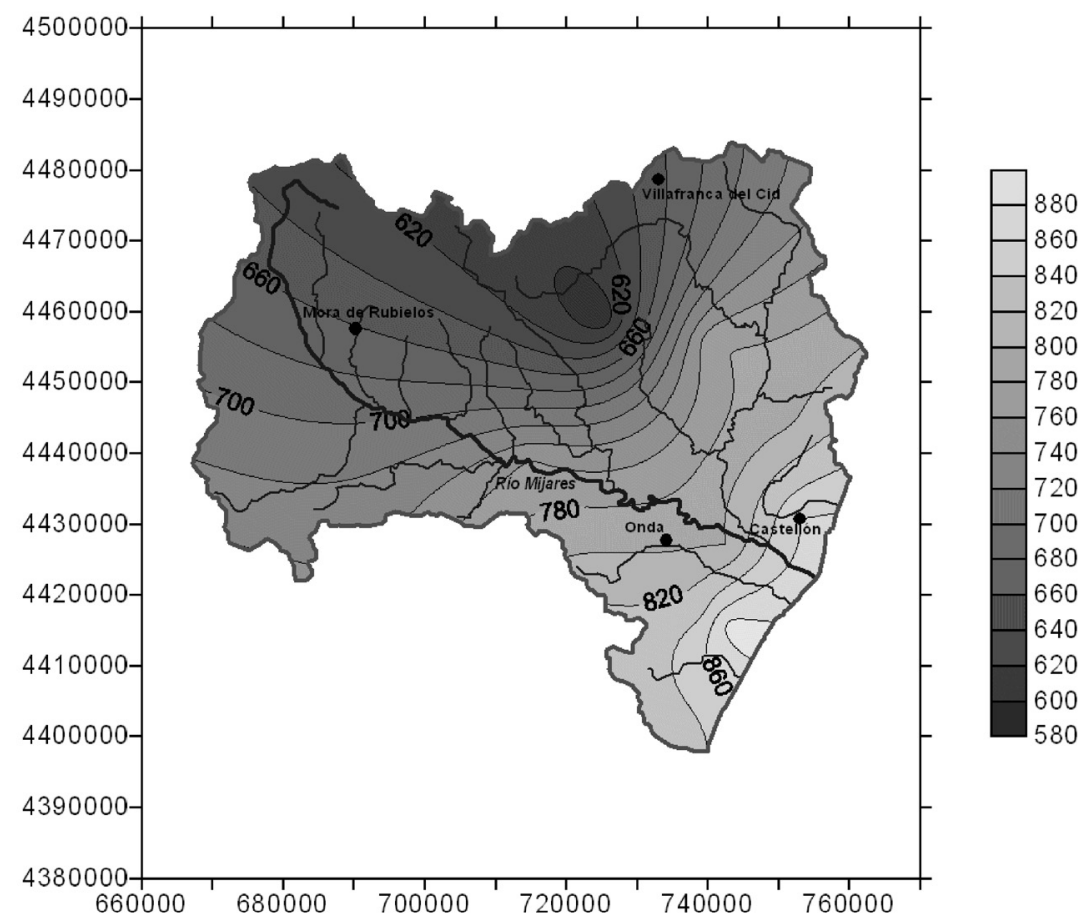

FIgURA 9. Valores medios anuales de la ETP según Thornthwaite (1950-2000).

Sin embargo, las cantidades de agua disponibles en el suelo no son siempre suficientes para que las evapotranspiraciones alcancen el valor total potencial exigido por la atmósfera:

$$
\mathrm{ETR} \leq \mathrm{ETP}
$$


Las diferencias son más acusadas a medida que el territorio es más seco. Estas diferencias llegan a superar el 50\% en gran parte de nuestra región. El valor medio es del 67,5\% de la ETP, (712/480 mm). De este modo, la ETR viene, pues, a representar el valor real de las pérdidas de agua por evapotranspiraciones. La diferencia entre las precipitaciones y las evapotranspiraciones reales representa el valor de la escorrentía total o recursos hídricos disponibles.

El valor de la ETR ha sido calculado igualmente mediante los métodos de Tornthwaite y de Turc. El valor de Thornthwaite ha sido deducido de los valores mensuales a través de las fichas hídricas. Los resultados medios anuales deducidos de las fichas hídricas de Thornthwaite han sido referenciados con los obtenidos aplicando la formulación de Turc para el cálculo medio anual de la ETR en cada observatorio:

$$
\mathrm{ETR}=\frac{P}{\sqrt{0^{\prime} 9+\frac{P^{2}}{L^{2}}}}
$$

siendo ETR: la evapotranspiración total anual media $(\mathrm{mm})$; P: la precipitación anual media $(\mathrm{mm})$; T: la temperatura anual media $\left({ }^{\circ} \mathrm{C}\right) \mathrm{y} \mathrm{L}:=300+25 \mathrm{~T}+0{ }^{\prime} 05 \mathrm{~T}^{3}$

Este método analítico de Turc, aplicado a cuencas de alta insolación, tiende a subestimar los valores de la evapotranspiración real (ETR) con respecto a los valores deducidos de las fichas hídricas de Thornthwaite. La función de ajuste que correlaciona ambas evaluaciones de la ETR aparece en la figura 10.

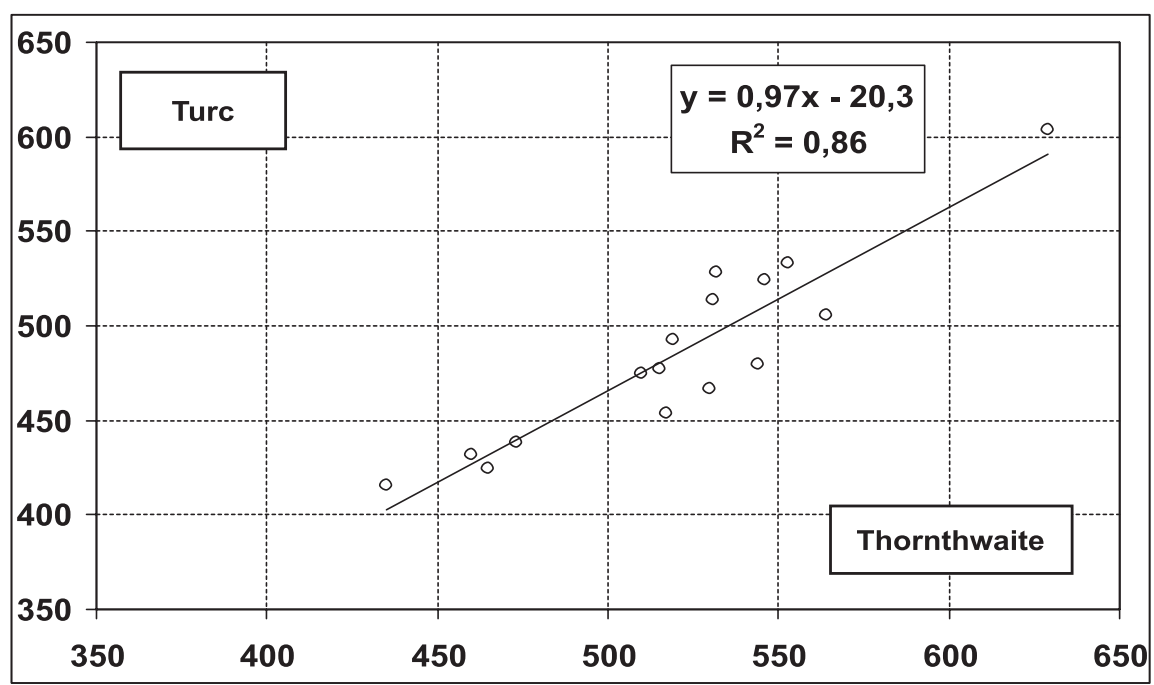

Figura 10. Función de ajuste entre la ETR $(\mathrm{mm})$ según el método de Thornthwaite y el método de Turc.

No obstante, los resultados obtenidos mediante la construcción de las fichas hídricas o de potencial agrícola con la ETP de Turc, en los escasos observatorios regionales tratables, han mostrado que la ETR anual era muy aproximadamente un 7-9 \% más elevada que la obtenida mediante la aplicación de la fórmula global de ETR (F. ELÍAS CASTILLO y F. 
CASTELLVI SENTIS, 1996; J. QUEREDA, 2009) y a la obtenida en las fichas hídricas de Thorntwaite. Este efecto estaría producido por la compensación hídrica post-estival de la reserva materializada en las fichas hídricas y no estimada en la formulación anual. En consecuencia, hemos ajustado la fórmula global de Turc para el cálculo de la ETR. Ello se ha realizado mediante la aplicación del coeficiente experimental 1,1 (Fig. 11). Así, la formulación aplicada ha sido:

$$
\mathrm{ETR}=1^{\prime} 1\left(\frac{P}{\sqrt{0,9+\left(\frac{P}{300+25 * T+0^{\prime} 05 * T^{3}}\right)^{2}}}\right)
$$

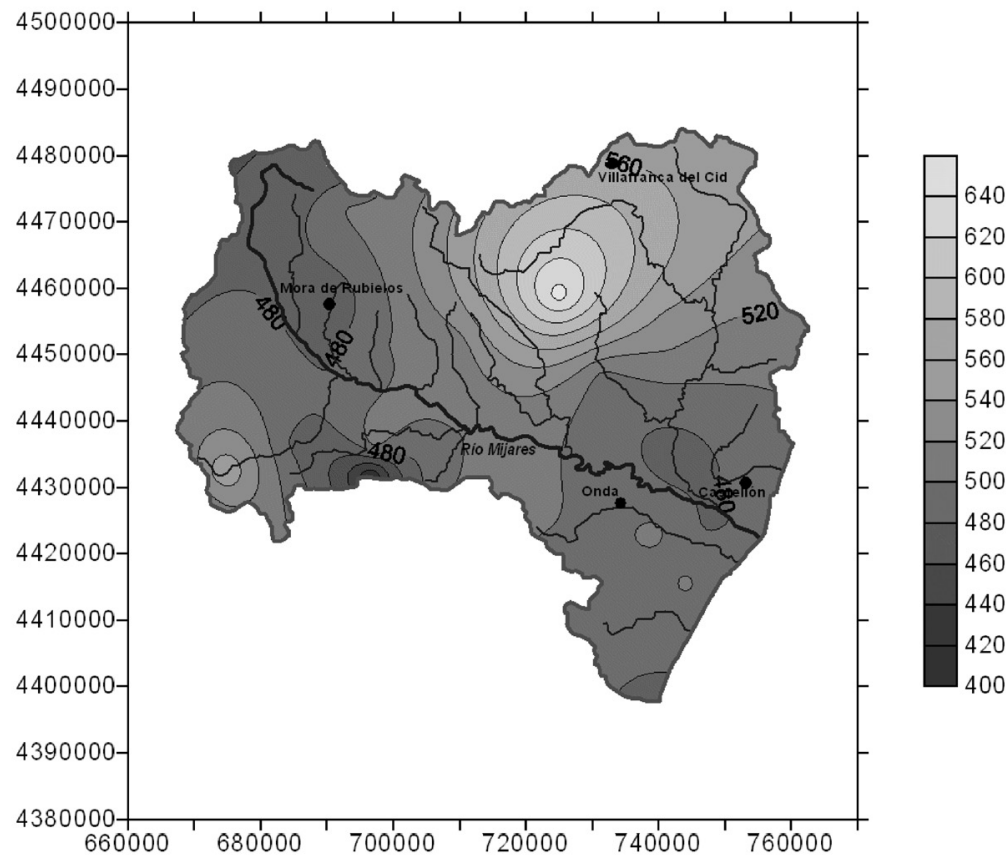

FIgURA 11. ETR media anual de Turc $(\mathrm{mm})$ sobre el Sistema Hidrográfico del río MijaresLa Plana (1950-2000). El valor medio anual es de $480 \mathrm{~mm}$.

El cálculo de la escorrentía. La evaluación de la escorrentía media anual ha sido efectuada con la metodología de Turc. Este método analítico, si bien mantiene la misma dependencia de la temperatura que el método de Thornthwaite utilizado en la ETP/ETR, integra en su formulación el valor de las precipitaciones para hallar la escorrentía media total anual o recursos hídricos disponibles. Un método que, al utilizar la precipitación (P) y la temperatura $(\mathrm{T})$, permite proyecciones basadas en la alteración de esos elementos. Este método analítico, aplicable a los distintos observatorios termopluviométricos, está basado en la siguiente ecuación: 


$$
\mathrm{A}=\mathrm{P}-\frac{P}{\sqrt{0,9+\left(\frac{P}{300+25^{*} T+0^{\prime} 05^{*} T^{3}}\right)^{2}}}
$$

Siendo A: la escorrentía total media anual $(\mathrm{mm})$; P: la precipitación media anual ( $\mathrm{mm})$ y T: la temperatura media anual $\left({ }^{\circ} \mathrm{C}\right)$.

De acuerdo al ajuste realizado en el cálculo de la ETR, la formulación aplicada ha sido:

$$
\mathrm{A}=\mathrm{P}-1^{\prime} 1\left(\frac{P}{\sqrt{0,9+\left(\frac{P}{300+25 * T+00^{\prime} 05 * T^{3}}\right)^{2}}}\right)
$$

El sistema hidrográfico del Mijares-La Plana dispone, de acuerdo con esta formulación, de una escorrentía media de $76 \mathrm{~mm}$. Los valores obtenidos sobre los puntos de observación meteorológica han sido proyectados cartográficamente mediante MDT. El modelo utilizado aquí ha sido de naturaleza teselar o raster de puntos georeferenciados distribuidos regularmente sobre el territorio. La interpolación de los valores calculados sobre el territorio sin observaciones ha sido el Kriging con función de correlaciones espaciales aplicadas mediante el programa Surfer. Finalmente, la obtención de los valores promedios regionales ha sido calculada mediante la computación de matrices o álgebra de mapas efectuada a través del SIG-IDRISI.

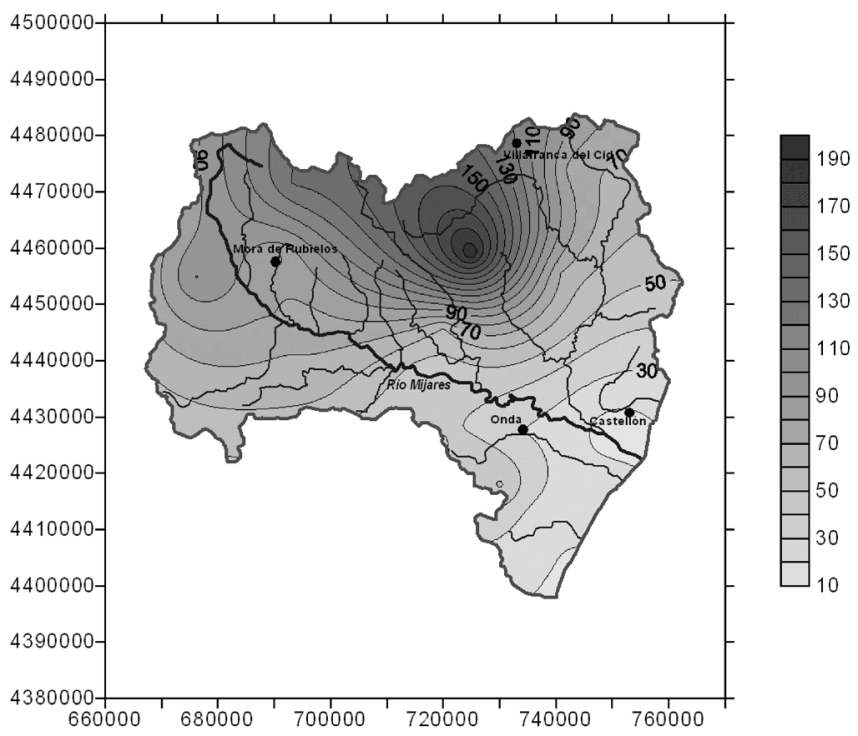

Figura 12. Escorrentías medias anuales sobre el Sistema Hidrográfico del río Mijares-La Plana (1950-2000). El valor medio anual es de $76 \mathrm{~mm}$. 
Tales valores son los que figuran en la base cartográfica elaborada como mapa de la escorrentía total media anual en mm sobre el territorio de la cuenca del Mijares-La Plana. Un mapa que viene a mostrar la limitación de los recursos hídricos disponibles sobre la cuenca. En su mayor parte, el territorio está por debajo de los $80 \mathrm{~mm}$ de escorrentía media anual y tan sólo se sobrepasa claramente dicho valor en el nudo ibérico del macizo de Penyagolosa $(1.813 \mathrm{~m})$. Los mismos coeficientes regionales de escorrentía, en las pequeñas cuencas de cabecera del Mijares (El Terde), Alfambra y Cenia, se hallan entre 0,10 y 0,12 (AFOROS, 1970; CHJ, 2000). Unos valores que se corresponden con el mapa de escorrentía resultante. La figura 12 muestra la proyección de los valores de la escorrentía media anual. Globalmente la escorrentía media de $76 \mathrm{~mm}$ supone una aportación media anual de $368 \mathrm{Hm}^{3} /$ año sobre los $4.846 \mathrm{Km}^{2}$ de la superficie total del Sistema Hidrográfico Mijares-La Plana. Una aportación muy ajustada para atender una demanda de $350 \mathrm{Hm}^{3} /$ año previstas en el PHN para el año 2.020, con el aumento demográfico experimentado.

\section{Necesidades hídricas presentes}

Los resultados obtenidos en nuestro análisis del ciclo hidrológico sobre la cuenca y sistema hidrográfico del río Mijares, y con las cautelas que cualquier metodología debe adoptar ante la insuficiencia de nuestras redes de medida e información, permiten establecer un balance hídrico. Las magnitudes agregadas sitúan unas aportaciones o recursos disponibles de $368 \mathrm{Hm}^{3} /$ año. Una aportación verdaderamente escasa, ya que representa tan sólo un $14 \%$ del volumen total precipitado sobre la cuenca y muy lejos del $30 \%$ promedio español. Frente a estos recursos propios se alzan unas demandas que, con el incremento demográfico experimentado, se sitúan en $350 \mathrm{Hm}^{3} /$ año (PHN, 2001). De este modo, el Sistema Hidrográfico Mijares-La Plana se situaría en una zona de escasez coyuntural muy vulnerable a cualquier cambio climático.

NIVEL DE LAS DEMANDAS EN LOS HORIZONTES DE 2010 Y 2020 (PHN) DEMANDAS DE AGUA PREVISTAS EN LA CUENCA DEL MIJARES

\begin{tabular}{|c|c|c|c|c|c|c|c|c|c|}
\hline \multicolumn{3}{|c|}{$1^{\text {er }}$ Horizonte } & \multicolumn{5}{c|}{$2^{\circ}$ Horizonte } \\
\hline URBANA & AGRÍCOLA & INDUST. & ECOLO & TOTAL & URBANA & AGRÍCOLA & INDUST. & ECOLO & TOTAL \\
\hline 50,95 & 244,50 & 18,00 & 15,00 & 328,45 & 57,21 & 244,50 & 21,00 & 15,00 & 337,71 \\
\hline
\end{tabular}

Este balance anuncia prematuramente la situación prevista en la reciente planificación hidrológica. En efecto la agregación de los recursos hídricos disponibles permite definir a la Cuenca del Mijares y su Sistema Hidrográfico como territorio en situación de encrucijada entre la cuenca excedentaria del Ebro y la deficitaria del Jucar. En cualquier caso, por sumarios e imperfectos que sean estos inventarios (A. GIL OLCINA, 1995) reflejan con absoluta nitidez la preocupante situación que ya, a nivel del año 2010, se nos ofrece. Todo un inquietante escenario, que todavía podría ser más alarmante ante la posibilidad de que las severas sequías padecidas en la última década puedan estar constituyendo una señal de alerta ante un cambio climático (J. QUEREDA, et al., 2009).

En este orden de previsiones, las bases científicas de los dos últimos informes Third and Fourth Assessment del IPCC (Panel Intergubernamental para el Cambio Climático), 
Climate Change, 2002 y 2007 de las Naciones Unidas, así como en la reciente Convención del IPCC Valencia (2007), basadas en los modelos del Hadley Centre, establecen que nuestra región mediterránea será la más vulnerable de Europa a los efectos del cambio climático. Vulnerabilidad basada en que, simultáneamente al aumento térmico, se produciría una reducción de recursos hídricos. El valor de esta reducción, en el modelo de tercera generación (HadCM3), podría alcanzar hasta un $25 \%$ de las actuales magnitudes de escorrentía regionales ya de por sí escasas, con valores medios anuales de $73 \mathrm{~mm}$ en las tierras de la Cuenca del Jucar (J. QUEREDA, E. MONTÓN y J. ESCRIG, 2009).

\section{La proyección de los recursos hídricos en el horizonte de los años 2025-2030}

El cambio de escenario atmosférico suscita de inmediato una preocupación por el impacto que tales variaciones climáticas puedan tener sobre los recursos hídricos. En efecto, la modificación de las condiciones atmosféricas actuales induciría una alteración del ciclo hidrológico natural y consecuentemente en los recursos hídricos naturales. Toda variación climática tendría su traducción inmediata en los valores de la escorrentía total anual media. En este sentido, las sequías pluviométricas podrían venir agravadas además por un crecimiento gradual de las evapotranspiraciones e intensificación del ciclo hidrológico.

Precisamente, tal y como hemos venido sosteniendo, esta concepción del ciclo hidrológico permite poder realizar una evaluación del impacto que las variaciones climáticas podrían tener sobre los recursos hídricos de la cuenca. La misma formulación que fundamenta nuestro análisis de la escorrentía actual a través del criterio de Turc, permite proyectar los impactos que sobre los recursos hídricos de la zona podría tener una alteración de los elementos climáticos.

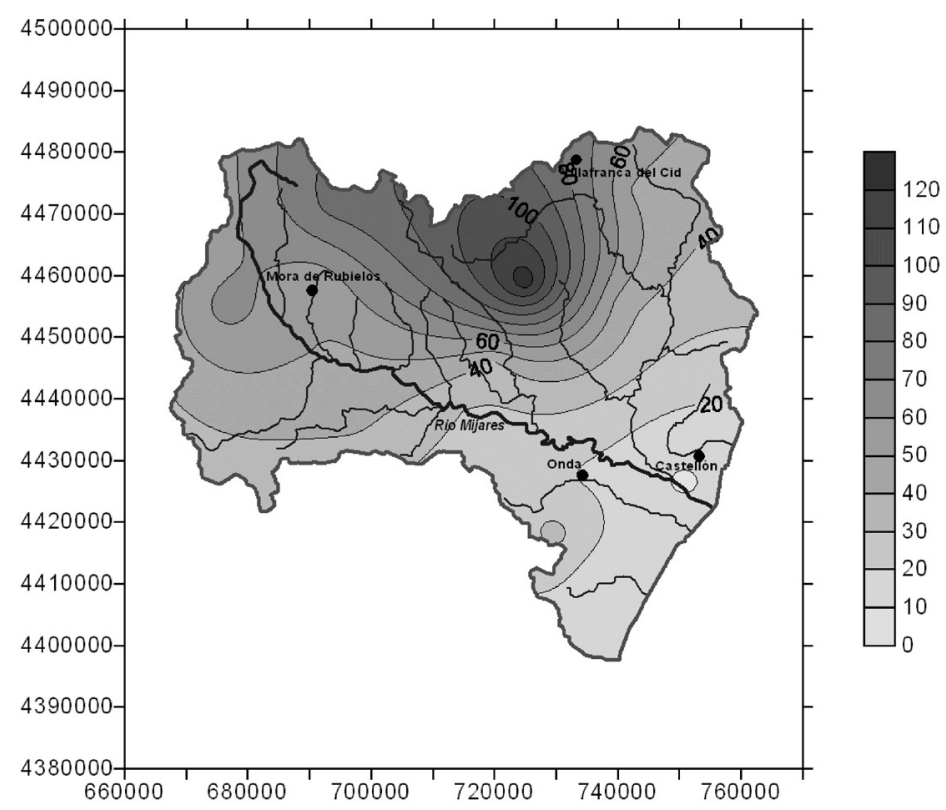

Figura 13. Escorrentía global (mm) del Sistema Hidrográfico del río Mijares-La Plana bajo un escenario climático configurado por un aumento térmico de $+1^{\circ} \mathrm{C}$ y una reducción de las precipitaciones del $5 \%$. 
En este orden de proyecciones, a tenor de los valores de tendencia climática regional registrados en el período 1950-2008 (J. QUEREDA, E. MONTÓN y J. ESCRIG, 2009), creemos que una buena ponderación de escenario climático previsible sobre la Cuenca del Júcar, en el horizonte del año 2030, puede venir encuadrada por un umbral máximo. Este escenario posible por impacto de un cambio climático, podría situarse en un aumento térmico de $1^{\circ} \mathrm{C}$ y un $5 \%$ menos de precipitaciones medias. Bajo estas condiciones climáticas, la reducción de recursos hídricos sería de gran severidad.

Esta reducción nos situaría ante una escorrentía de tan sólo 49 mm, un 35 \% inferior a la actual $(76 \mathrm{~mm})$. Esta escorrentía, sobre los $4.846 \mathrm{Km}^{2}$ de la superficie total del Sistema Hidrográfico Mijares-La Plana, arrojaría una disponibilidad de agua de tan sólo $237 \mathrm{H}^{3}$ / año (Fig. 13). Esta disponibilidad de recursos hídricos sería netamente insuficiente para cubrir los $350 \mathrm{Hm}^{3}$ demandados en el horizonte del año 2020, según lo previsto en el Plan de Cuenca del Júcar (CHJ 1999).

\section{Conclusiones}

El objetivo del presente estudio ha sido analizar y calcular cuanta agua hay sobre la cuenca del Mijares, así como proyectar los impactos que un cambio climático podría tener sobre esos mismos recursos hídricos. Este objetivo ha sido considerado bajo la concepción del ciclo hidrológico. En este concepto, los recursos naturales propios son los que se generan a partir de la precipitación y que, subsistentes a la evapotranspiración, nutren y son aportados a través de las escorrentías superficiales directas y de la recarga de los acuíferos. Un sistema que desemboca en un balance de agua expresado por el concepto clave de la escorrentía (E).

Ante las dificultades de cálculo que plantea la escasa red de las estaciones de aforo, hemos aplicado una metodología finalista basada en la red meteorológica. Una metodología que tiende a evaluar los recursos hídricos mediante la modelización de los elementos climáticos básicos que intervienen en el ciclo del agua. Dadas las estrechas relaciones existentes entre los elementos climáticos que intervienen en el ciclo hidrológico natural, es posible establecer funciones de ajuste que vinculen las escorrentías totales con las precipitaciones y con los valores de $\mathrm{P}$ y $\mathrm{T}$.

Este método permite alcanzar buenos resultados. Incluso por definición, estos resultados pueden ser óptimos sobre la base de reunir dos condiciones. La primera es que la red meteorológica disponible, suficiente en cantidad y calidad, no presente sesgos de localización que falseen la modelización cartográfica posterior. La segunda es la medida rigurosa de la evapotranspiración. Condiciones que han sido debidamente consideradas en nuestro análisis. La ETP-ETR ha sido evaluada con la metodología de Tornthwaite y de Turc, las más apropiadas a los datos existentes. La escorrentía resultante, ha sido contrastada con la obtenida a través de la aplicación de Turc. Ello ha permitido ajustar la formulación de Turc mediante una ligera modificación del efecto de compensación hídrica post-estival. Esta formulación, basada en la relación P y T, ha permitido calcular la gran afección que sobre los recursos hídricos podría tener un cambio climático. Así, bajo un escenario climático con un aumento de $1^{\circ} \mathrm{C}$ sobre las temperaturas actuales y una disminución de un $5 \%$ de las precipitaciones actuales, el Sistema Hidrográfico del Mijares-La Plana reduciría su escorrentía en un $35 \%$.

\section{Referencias bibliográficas}

AFOROS, (1970). Anuario, 8 Cuenca del Jugar, Ministerio de Obras Públicas, Dirección General de Obras Hidráulicas. 
ALMARZA, C. (1984). Fichas hídricas normalizadas y otros parámetros hidrometeorológicos, INM, 3 vols.

ANDRÉ, J-C., ROYER, J., (1999) «Fluctuations à court terme du climat et l'interpretation des observations recentes en termes d'effet de serre», La Météorologie, 8a, série, 28, 12-22.

AYALA-CARCEDO, J. e IGLESIAS, A. (2000). Impactos del posible cambio climático sobre los recursos hídricos, el diseño y la planificación hidrológica en la España Penínsular, BBVA, Servicio de Estudios.

BROCHET, P. et al. (1975). Contribution a l'étude agrométéorologique du mais. Monographie de la $\mathrm{MN}, \mathrm{n}^{\circ} 95$.

BROCHET, P. et al. (1977). Etude agrométéorologique du blé tendre d'hiver. Monographie de la $\mathrm{MN}, \mathrm{n}^{\circ} 101$.

CIID (1977). La Météorologie, Número especial «Evapotranspiration», VI, 11, 1977, 165 pp.

CONSELLERÍA D'OBRES PUBLIQUES, URBANISME I TRANSPORTS (GENERALITAT VALENCIANA). Libro Blanco del Agua en la Comunidad Valenciana. Situación actual y bases para una nueva política hidráulica. Valencia, 1985.

CONFEDERACIÓN HIDROGRÁFICA DEL JÚCAR (1992) Plan Hidrológico. Proyecto de Directrices, $191 \mathrm{pp}$.

CONFEDERACIÓN HIDROGRÁFICA DEL JÚCAR (1999). Plan Hidrológico de Cuenca del Júcar, 32 pp. (BOE, 27de agosto, 1999).

ELIAS CASTILLO, F. y CASTELLVI SENTIS, F. (1996). Agrometeorología, Ministerio de Agricultura, Pesca y Alimentación, 516 pp.

ESCRIG BARBERA, J. (1985). El espacio rural de Atzeneta. Colegio Universitario de Castellón, $194 \mathrm{pp}$.

GIL OLCINA, A. (1989). «Causas climáticas de las riadas». Avenidas fluviales e inundaciones en la Cuenca del Mediterráneo, Instituto Universitario de Geografía, Universidad de Alicante, pp. 15-30.

IPCC Working Group I Four Assessment Report (2007). Climate Change 2001: The Scientific Basis. Draft version in http://www.gcrio.org/online.html.

LÓPEZ GÓMEZ, A. (1957). «Evolución agraria de la Plana de Castellón». Estudios Geográficos, XVIII, págs. 309-360.

MIMAM (2000). El Libro Blanco del Agua en España, Madrid, Ministerio de Medio Ambiente, 637 pp.

MIMAM (2001). Plan Hidrológico Nacional, Madrid, Ministerio de Medio Ambiente, 5 vols.

MIMAM (2007). PNACC, Plan Nacional de Adaptación al Cambio Climático. MIMAM.

MONTÓN CHIVA, E.y QUEREDA SALA, J. (1997). ¿Hacia un cambio climático?: Las tendencias del clima en la cuenca occidental del Mediterráneo desde mediados del siglo XIX. Fundación Dávalos Fletcher. Castellón. 520 pp.

OLCINA CANTOS, J. (1993). Episodios meteorológicos de consecuencias catastróficas en tierras alicantinas, Tesis doctoral, 3 vols, Universidad de Alicante.

PNACC (2007). Plan Nacional de Adaptación al Cambio Climático. MIMAM.

QUEREDA SALA, J. (1976). El clima de la Provincia de Castellón. Dip. Provincial, 133 pp.

QUEREDA SALA, J. y CALVO CASES, A. (1981). «El ciclo del agua y su importancia», Millars $V I I$, pp. 25-48.

QUEREDA SALA, J. (1982). Castellón, precipitaciones y radiación solar, Excmo. Ayuntamiento de Castellón, 40 pp.

QUEREDA SALA, J. (1983).» Recursos pluviométricos y exigencias evaporativas en la Provincia de Castellón». Boletín Credicoop, pp.34-41.

QUEREDA SALA, J. y MORELL EVANGELISTA, I. (1983).» Los recursos hídricos y su balance en la Provincia de Castellón». BSCC, LIX, pp. 201-218.

QUEREDA SALA, J. (1990). «Intense Cyclones in the Spanish Mediterranean». Weather, Royal Meteorological Society, 45, 7, pp. 278-279.

QUEREDA SALA, J. (1990). «Interactions mer-atmosphere et cyclogenèse en Mediterranée». Météorologie Maritime, 146, 1, pp. 26-30.

QUEREDA SALA, J., MONTÓN CHIVA, E. y ESCRIG BARBERA, J. (2009). Evaluación del cambio climático y de su impacto sobre los recursos hídricos en la Cuenca del Júcar. GV-Fundación Agua y Progreso, 165 pp. 
QUEREDA, J. (2009). Curso de Climatología General, Colecc. Universitas, 280 pp.

THORNTHWAITE, C.W. (1948). «An approach toward a rational classification of climate». Geographical Review: 55-94.

THORNTHWAITE, C.W. (1948). «Micrometeorology of the surface layer of the atmosphere». Public. Climat. 1 bis, 5.

THORNTHWAITE, C.W. and MATHER, J.R. (1955). «The water balance». Publications in Climatology 8 (1).Laboratory of Climatology. Centerton, N.J.

TURC, L. (1954). « Le bilan d'eau des sols : Relations entre les Precipitations, l'Evapotranspiration et l'Ecoulement », Annales Agronomiques, pp. 491-595.

TURC, L. (1961). «Evaluation des besoins en eau d'irrigation, evapotranspiration potencielle». Annales Agronomiques, pp.13-49. 